\title{
DESIGN CONSIDERATIONS FOR MEDICAL PROTON ACCELERATORS
}

\author{
George Coutrakon, Ph.D., James M. Slater, M.D., F.A.C.R., \\ Abiel Ghebremedhin, Ph.D. Loma Linda University Medical Center 11234 Anderson Street \\ Loma Linda, CA 92354, e-mail: coutrak@proton.llumc.edu
}

\begin{abstract}
The design requirements for current heavy-particle accelerators operated within a hospital to deliver radiation therapy must satisfy both clinical and research needs. Advances in dedicated beam delivery systems for clinical utilization and biological studies add requirements that previous accelerators did not have. Eight years experience using the Loma Linda University proton facility has emphasized that the most important requirements are safety, reliability, beam stability, low energy consumption, and efficiency of beam delivery to the treatment rooms. In the future, raster scanning techniques will add further demands on the control of beam energy, intensity, and position stability. Rapid and precise flexibility in changing beam parameters is essential for satisfying clinical needs; electronic rather than mechanical control is clearly preferable for clinical use. Biological research increases the need to expand the margins of some clinical requirements, such as beam size, intensity, and energy ranges. Both clinical and research activities require a totally integrated control system, beginning with the ion source and continuing through the accelerator and switchyard to multiple rooms and each beam delivery system therein. Accordingly, designing the clinical accelerator requires a highly orchestrated design effort, involving the entire facility. Detailed design requirements addressing these issues will be presented.
\end{abstract}

\section{INTRODUCTION}

More than forty years have passed since protons were first used in treating localized tumors in humans. In the meantime, twenty facilities worldwide have treated more than 23,000 patients with proton beams. ${ }^{1}$ Until 1990, all of these facilities used previously existing accelerators, which were designed for physics research and later modified for medical use. From 1987 to 1990, the first medical proton accelerator was designed and built for the Loma Linda University Proton Accelerator Facility ${ }^{2}$ (LLUPAF) in Loma Linda, CA. This project was a cooperative effort between Loma Linda University, Fermi National Accelerator Laboratory (Fermilab), and Science Applications International Corporation (SAIC). Since 1990, over four thousand patients have been treated with protons at LLUPAF. To date, four more hospital-based facilities are under construction: one in Boston, MA and three in Japan. In addition, many new facilities are in the planning stages in the US, Europe, and Japan.
The advantages of a hospital-based facility over a laboratory-based facility are great. In a hospital, health care professionals can work as a team more efficiently and more effectively in planning, preparing and executing high precision radiotherapy treatments for each patient. In addition, the expensive ancillary equipment (such as CT) and hospital staff are already in place for patient preparation and treatment. Beam time can be dedicated for medical use, which is not the case at physics research laboratories. Finally, anesthetized and other nonambulatory patients can be treated only in a hospital setting. In many of the new facilities, basic research in radiation biology will also need to be included in the facility requirements as well. In this paper, we examine the primary requirements that any new proton or light ion accelerator will face in a hospital or clinical setting.

\section{PROTON DOSIMETRY}

The primary advantage of protons over therapeutic x-rays is shown in dose vs. penetration data (Fig.1). One sees that protons give a higher dose at deeper sites and a lower dose near the entrance to the body, where healthy tissue is likely to be. Unlike x-rays, protons have no "exit dose," which spares healthy tissue beyond the Bragg peak.

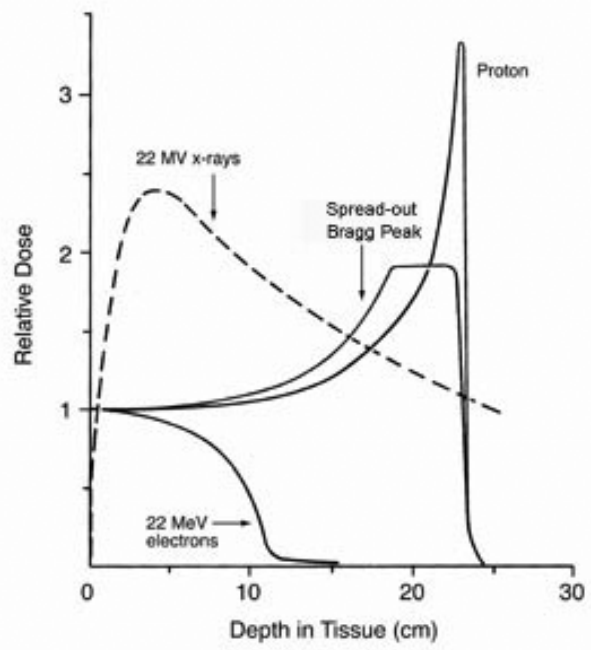

Fig. 1: Dose vs. depth for therapeutic proton, x-ray, and electron beams. The mono-energetic Bragg peak is the narrow one on the right. The spread-out Bragg peak (SOBP) is generated from mono-energetic peaks as shown in Fig. 2. The maximum depth of beam penetration and width of the SOBP can be controlled by the beam energy.

The energy of the proton beam can be so adjusted from the accelerator that protons penetrate to the correct depth for each tumor. By adding Bragg peaks of successively lower 
energies and intensities (Fig. 2) a SOBP can be generated which has uniform dose over the entire depth of the tumor. The energy accuracy for each Bragg peak is quite precise and dictates the energy accuracy requirement for the accelerator, as is discussed later. The ability to achieve dose uniformity in depth as well as transverse to the beam direction within the tumor volume is a primary requirement in all radiotherapy treatments.

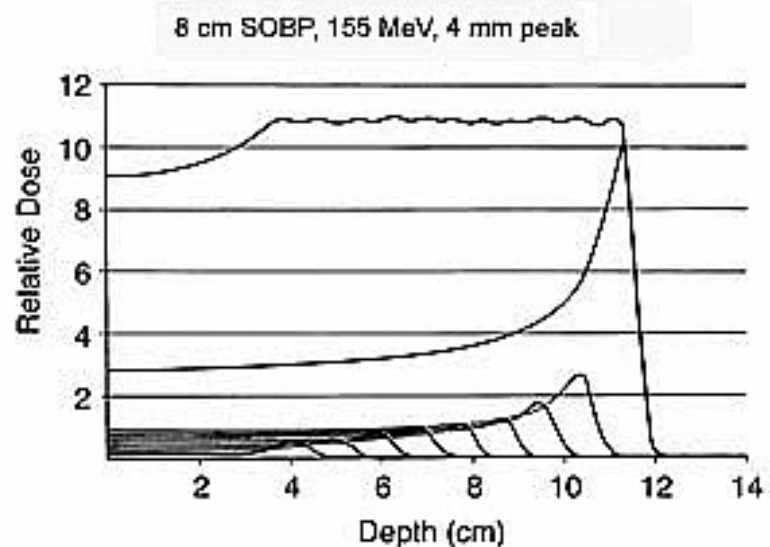

Fig.2: A spread-out Bragg peak (SOBP) generated from the addition of mono-energetic Bragg peaks. The dose uniformity in the SOBP between $4 \mathrm{~cm}$ and $12 \mathrm{~cm}$ is less than $+/-2 \%$ with no energy or intensity errors.

\section{ACCELERATOR SYSTEM REQUIREMENTS}

The primary function of any proton therapy accelerator is to provide treatment rooms with a specified energy and intensity (or dose rate) throughout each treatment. In addition, accelerator or beam transport magnets must be capable of terminating beam to within $1 \%$ of the prescribed dose for each treatment. The emittance from the accelerator should be as small as possible, to allow use of smaller magnet apertures and smaller magnets in the beam transport lines and rotating gantries. Typical emittance values for several classes of accelerators are presented in Table 2. The accuracy requirements for energy and intensity will depend largely on the type of beam delivery system that the treatment room uses. For example, scanning the target volume with a narrow pencil beam and rapid energy changes from the accelerator will have much tighter accelerator energy, intensity, and spot position requirements than a beam delivery system that uses lead foils to passively enlarge the beam. Of course, active scanning will offer superior sparing of normal tissues and is a clear goal for all proton and light ion facilities. Examples of active and passive beam delivery systems are shown in Fig. 3a and 3b. For active beam scanning, the accelerator control system must contain a library of energy ramp sets that can be executed in rapid succession to generate SOBPs (Fig.2) at the patient. The exact sequence of energies and intensities must be transmitted electronically from the treatment planning computer for each patient.

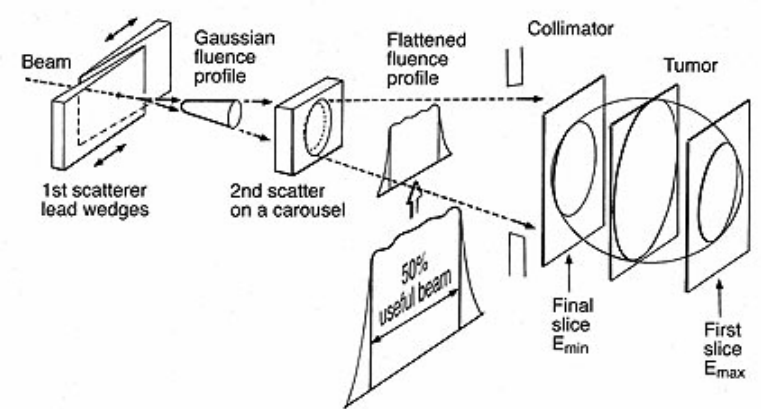

Fig. 3a: Lead foil scattering system to enlarge the beam laterally after transport to the treatment room. Oftentimes, another passive device (not shown) in the treatment room, called a range modulator, is used to degrade parts of the beam to various ranges, which generate the SOBP. This is used in lieu of multiple energy changes from the accelerator. The time structure of the extracted beam is not important in this case.

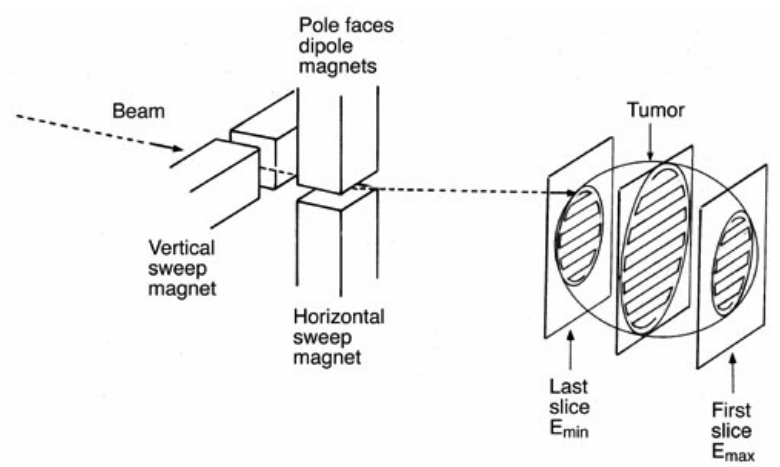

Fig. 3b: Raster scan magnet system to sweep a small $(1 \mathrm{~cm})$ beam across the tumor volume. Here, the energy of the beam must be varied from the accelerator.

Fig. 4a and 4b show the ranges of energy and intensity that are required for proton therapy. The energy range most often specified for a therapy accelerator is 70 to 250 $\mathrm{MeV}$, which corresponds to 3 to $40 \mathrm{~cm}$ range in water or soft tissue. Fig. $4 \mathrm{~b}$ shows the fluence requirements in units of protons $/ \mathrm{cm}^{2} / \mathrm{Gy}$ and should be scaled using the appropriate field size, dose rate, and modulation depth that is desired. The ideal facility should have efficient beam extraction and beam delivery systems which will require the minimum beam current (protons/min) to achieve treatment times below several minutes with doses of approximately 2 Gray. This minimizes the risk of an inadvertent overdose to the patient.

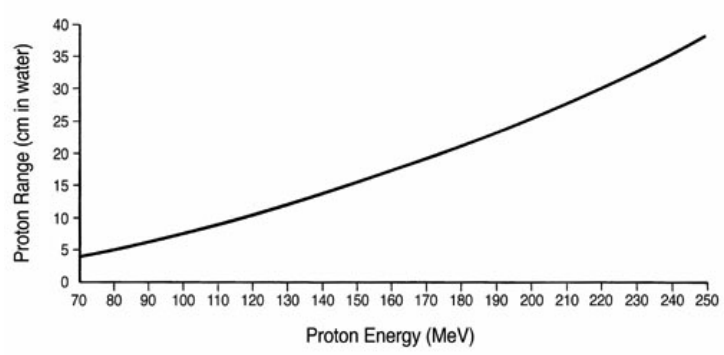

Fig. 4a: Proton energy vs. range in water ${ }^{3}$ 


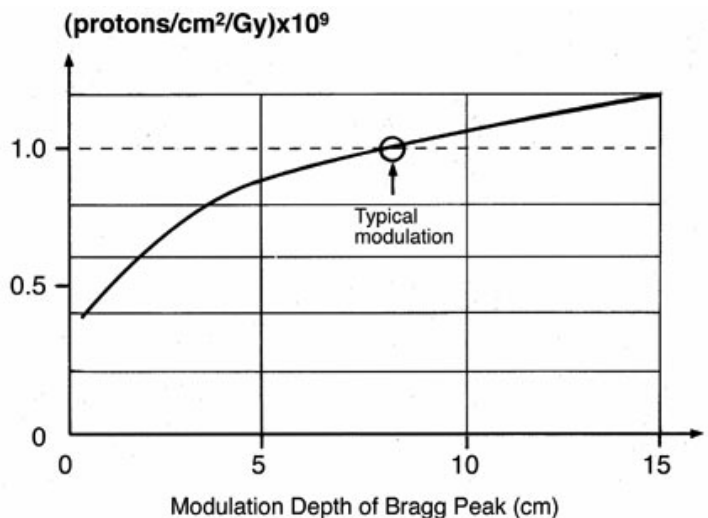

Fig. 4b: Proton fluence/Gray versus width of SOBP for $155 \mathrm{MeV}$ maximum energy ${ }^{4}$. The proton fluence per Gray for $250 \mathrm{MeV}$ maximum energy is about $30 \%$ higher than this curve and about $30 \%$ less for SOBPs with $100 \mathrm{MeV}$ maximum energy.

\section{GENERAL REQUIREMENTS}

Some obvious requirements only need mentioning for completeness, but require careful attention for a successful facility. Accelerator reliability is one of the top priorities for all therapy operations. For example, the LLUPAF synchrotron has enjoyed $98 \%$ uptime for patient treatments with five treatment days per week and 24-hour operations.

The proton synchrotron services four treatment rooms and one research room. The high reliability has been accomplished with the help of a maintenance team that monitors various systems to perform preventive maintenance before failures occur, particularly with magnet power supplies, vacuum components and water cooling systems. Accelerator reliability is extremely important, particularly for patients in the middle of threeor four-week treatment schedules. Clinical data have shown that patients whose treatments have been interrupted for two or more weeks have increased risk of tumor recurrence compared to patients who keep regularly scheduled treatments. Most of the accelerator down time (2\%) over the past three years was due to power outages that caused vacuum failures and magnet power supply problems related to chilled water systems. Oftentimes, any missed treatments can be made up on weekends or by increasing daily dose fractions.

A new facility must be designed for high patient throughput to be financially solvent and to rapidly acquire statistically significant clinical data for each disease site being treated. High patient throughput requires a large number of activities to run smoothly. On the accelerator end, the ability to switch beam energies and treatment room destinations quickly and reliably is crucial to reducing patient waiting times. For the same reason, the dose rate (or intensity) from the accelerator must also be sufficiently high to avoid limiting patient throughput.

At LLUPAF, the average treatment time per patient is about two minutes and the time to switch treatment rooms less than one minute. Since the time to align the patient requires typically ten to fifteen minutes, the accelerator performance does not currently limit the patient throughput. The Loma Linda facility has demonstrated patient throughput as high as 125 patients in a 16-hour day.

Attention should also be given to power supply stability in accelerator systems. This affects energy, intensity, and position stability of extracted beams. In beam transport lines, dipole bending magnet stability effects position stability of the beam in the treatment rooms. Typical values of beam position tolerance require fluctuations less than $\pm 1 \mathrm{~mm}$ in the treatment rooms, at the end of a beam line that may be 50 meters from the accelerator. In some cases, the current output from a bending magnet power supply in the beam lines may require current stability to the magnets better than $0.1 \%$. Reproducible and stable current output to all beam transport magnets is crucial to avoid beam losses during treatment and to maintain correct beam position in the treatment rooms.

Efficiency of beam extraction from the accelerator is an area where higher efficiency will lower the radiation activation of accelerator components and make serviceability much easier. Shielding requirements for personnel will also be lower. Higher beam transport efficiency through the switchyard will lower the required intensity from the accelerator, thereby reducing neutron exposure to personnel and the general public. Typical values for beam extraction efficiency and beam transport efficiency at LLUPAF are $90 \%$, with extracted beam currents less than $5 \mathrm{nA}$ (time averaged). This leads to very low equipment activation and low exposure to personnel.

\section{REQUIREMENTS FOR BIOLOGICAL STUDIES}

A large NASA program has begun at Loma Linda to investigate the health effects of space radiation on astronauts on long missions. These experiments present requirements that differ from therapy beam requirements. In general, they require very low dose exposures, extending from one day to several weeks. To accommodate these requirements, the accelerator must have intensity control that is roughly 100 times lower than therapy beams with energies from 20 to $300 \mathrm{MeV}$. Small energy changes $(<4 \mathrm{MeV})$ of once per second would be required to cover this large dynamic range. Many of these irradiations must be done between patient treatments and therefore require a separate room with semipermanent biologic setups. Accelerator and beam line controls must be able to switch between a treatment room and the research room fast enough (less than one minute) so that patient wait times are low. In addition, measurements of mammalian cell damage on the sharp distal edge falloff of the Bragg peak (see Fig. 1) require energy stability of $0.1 \%$ from the accelerator. Fortunately, this is within accuracy and stability limits of modern proton therapy accelerators. These are only two examples of a large class 
of research requirements that need to be satisfied by the accelerator and control system.

\section{INTENSITY REQUIREMENTS FOR BEAM SCANNING}

The stacked Bragg peaks in Fig. 2 show that the integrated dose for each peak is different. A dynamic range of 20:1 is required to obtain the desired dose uniformity throughout the SOBP. Approximately ten to twenty energies will be required for each treatment to generate the desired dose uniformity over the entire depth of the tumor. Studies have shown ${ }^{5}$ that it is much more time efficient to vary the accelerator intensity for different proton energies than to vary the dwell time for individual energies to achieve the 20:1 range of doses between Bragg peaks. Variable intensity control is, therefore, desirable. The ability to control the beam current from the accelerator with an accuracy of $\pm 10 \%$ over the $20: 1$ dynamic range also is desirable. For beam scanning applications, it is desirable to have the intensity as uniform as possible as the beam is swept across a target volume, to "paint" a uniform dose distribution. The detailed requirement depends on the beam spot size, $\sigma$, in the patient and the sweep speed, $v$, of the scanning magnets. In general, all frequencies below $\mathrm{f}=$ $\mathrm{v} / \sigma$ should have intensity ripple below $\pm 3 \%$ to have dose errors less than $\pm 3 \%$ in the plane transverse to the beam direction. For example, a typical beam diameter of 1 $\mathrm{cm}$ and magnet sweep speed of $1 \mathrm{~cm} / \mathrm{ms}$, yields an intensity stability requirement of $\pm 3 \%$ for frequencies below $1 \mathrm{kHz}$.

\section{ENERGY CONTROL REQUIREMENTS FOR BEAM SCANNING}

Due to the sharp falloff at the distal edge of a monoenergetic Bragg peak, each energy must be delivered very precisely relative to the preceding one to generate a superposition of Bragg peaks (or SOBP) with good dose uniformity throughout the depth of the target. When the mono-energetic beams are generated from the accelerator and not from passive devices in the treatment room beam delivery system, much tighter energy control is required. A minimum targeted dose uniformity throughout the tumor volume is currently $\pm 3 \%$. Note that the distal dose falloff is sharper at lower energies due to lower energy straggling for particles of less range in tissue. Therefore, the dose uniformity, in depth, is more sensitive to range errors at low energies than at high energies. The effects of a $0.7 \mathrm{MeV}$ error (or equivalently $1 \mathrm{~mm}$ of range) from one of the Bragg peaks can be clearly seen in Fig. 5, where the dose non-uniformity has increased to $10 \%$ near the distal edge. Further simulations have shown that an energy accuracy of $\pm 0.1 \mathrm{MeV}$ to $\pm 0.3 \mathrm{MeV}$ (see Table 1 , below) is necessary to insure $\pm 3 \%$ dose uniformity in the SOBP.

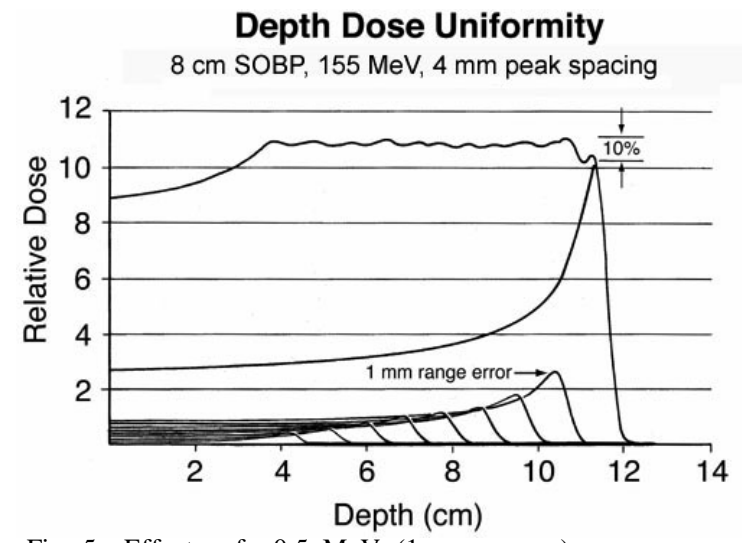

Fig. 5 Effects of $0.5 \mathrm{MeV}$ ( $1 \mathrm{~mm}$ range) energy error on the uniformity of the SOBP. Note that the clinical tolerance of $\pm 3 \%$ has been exceeded.

Table 1: Energy accuracy to achieve $\pm 3 \%$ Dose Uniformity in the SOBP

\begin{tabular}{|l|l|l|l|}
\hline $\begin{array}{l}\text { Energy } \\
\text { (maximum) }\end{array}$ & $\begin{array}{l}\text { Allowed } \\
\text { Energy } \\
\text { Error }\end{array}$ & $\begin{array}{l}\text { Correspondi } \\
\text { ng Range } \\
\text { Error }\end{array}$ & $\begin{array}{l}\text { Distal } \\
\text { Falloff of Bragg } \\
\text { peak } \\
\mathbf{1 0 \%})\end{array}$ \\
\hline $100 \mathrm{MeV}$ & $\pm 0.1 \mathrm{MeV}$ & $\pm 0.1 \mathrm{~mm}$ & $1.8 \mathrm{~mm}$ \\
\hline $155 \mathrm{MeV}$ & $\pm 0.2 \mathrm{MeV}$ & $\pm 0.4 \mathrm{~mm}$ & $4.3 \mathrm{~mm}$ \\
\hline $250 \mathrm{MeV}$ & $\pm 0.3 \mathrm{MeV}$ & $\pm 0.8 \mathrm{~mm}$ & $10.0 \mathrm{~mm}$ \\
\hline
\end{tabular}

Fig. 6 shows the dose uniformity as a function of range error for SOBPs with $100 \mathrm{MeV}, 155 \mathrm{MeV}$ and $250 \mathrm{MeV}$ maximum energies. The graph is a compilation of 1000 SOBPs with random range errors selected from a gaussian error distribution. The dose uniformity is indicated for the 95 percentile group of the 1000 SOBPs which were generated for each gaussian range error. The range error for $\pm 3 \%$ dose uniformity is consistent with the values shown in Table 1.

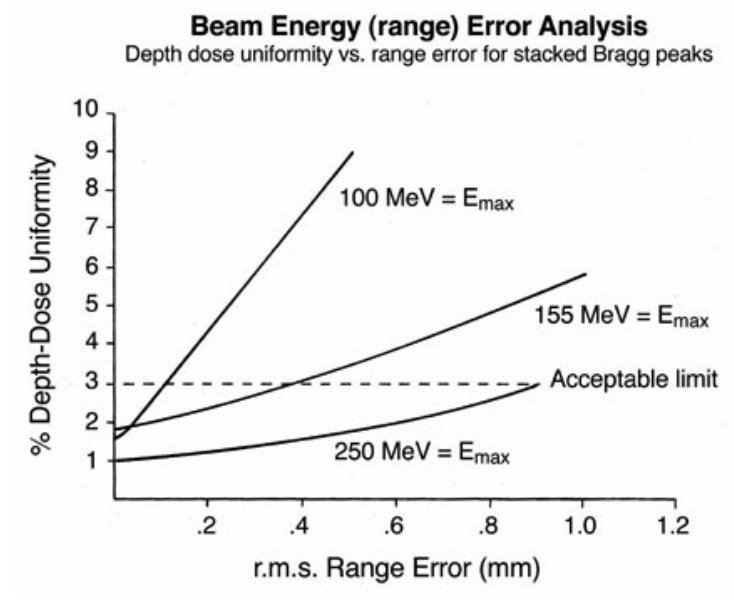

Fig. 6: A monte carlo analysis of 1000 SOBPs for each gaussian error function. Each mono-energetic Bragg peak is given an error and the uniformity of the SOBP is calculated. The curves indicate the upper limit of non-uniformity for $95 \%$ of the cases.

Comparison of the three basic accelerator types for proton therapy is outlined in Table 2. All three designs have been shown to work for therapy applications and all 
three are currently being built or used for therapy facilities around the world. The parameters listed above are those that customers examine most when selecting an accelerator. Vendors of proton therapy synchrotrons include Mitsubishi Electric Corp. in Kobe, Japan and Optivus Technology, Inc. of San Bernardino, CA. The intensity limit for synchrotrons in Table 2 is taken from the Hitachi accelerator, which uses multiturn injection.

The cost for each type of accelerator has not been included because it depends on the vendor's amortization schedule and the quantity of units to be sold. For example, the cost of development, engineering and overhead may be included differently among vendors. This dependence is probably larger than the difference between economical and more expensive designs. Roughly speaking, cost ranges can be expected to fall between eight and fifteen million dollars (in 1999 dollars) for the three accelerator types, with maximum energy capabilities between 220 to 270 $\mathrm{MeV}$.

Table 2: Accelerator comparisons

\begin{tabular}{|c|c|c|c|}
\hline & Synchrotron & Cyclotron & LINAC \\
\hline Vendor(s) & $\begin{array}{l}\text { Fermilab/ } \\
\text { Optivus } \\
\text { Technology } \\
\text { (USA) } \\
\text { Hitachi } \\
\text { (Japan) }\end{array}$ & $\begin{array}{l}\text { IBA } \\
\text { (Belgium) }\end{array}$ & ENEA(Italy) \\
\hline $\begin{array}{ll}\text { Energy } & \text { level } \\
\text { selection } & \end{array}$ & continuous & fixed" & continuous \\
\hline $\begin{array}{l}\text { Intensity limit } \\
\text { (ave.) }\end{array}$ & $5 \times 10^{12} / \mathrm{min}$. & AHAN & AHAN \\
\hline $\begin{array}{l}\text { Size (diam. or } \\
\text { length) }\end{array}$ & 6 meters & 4 meters & 37 meters \\
\hline $\begin{array}{l}\text { Ave. power } \\
\text { consumed } \\
\text { (beam on) }\end{array}$ & $370 \mathrm{~kW}$ & $300 \mathrm{~kW}$ & $320 \mathrm{~kW}$ \\
\hline $\begin{array}{l}\text { Beam emittance } \\
\text { (unnormalized, } \\
67 \% \text { of beam) }\end{array}$ & $\begin{array}{ll}1-3 & \pi \\
\mathrm{mrad} & \mathrm{mm}-\end{array}$ & $\begin{array}{l}10 \pi \quad \mathrm{mm}- \\
\mathrm{mrad}^{*}\end{array}$ & $\begin{array}{l}0.1 \pi \\
\mathrm{mm}-\mathrm{mrad}\end{array}$ \\
\hline $\begin{array}{ll}\text { Rapid } & \text { energy } \\
\text { changes }\end{array}$ & $4 \mathrm{MeV} / \mathrm{s}$ & $4 \mathrm{MeV} / \mathrm{s}$ & $4 \mathrm{MeV} / \mathrm{ms}$ \\
\hline $\begin{array}{l}\text { Duty factor (\% } \\
\text { beam on time) }\end{array}$ & $20 \%$ at $0.5 \mathrm{~Hz}$ & $\begin{array}{ll}100 \% & \text { or } \\
\mathrm{CW} & \\
\end{array}$ & $\begin{array}{l}0.1 \% \\
\text { at } 300 \mathrm{~Hz}\end{array}$ \\
\hline $\begin{array}{l}\text { Beam intensity } \\
\text { uniformity (for } \\
\text { scanned beams) }\end{array}$ & adequate $^{* * * *}$ & good & good \\
\hline $\begin{array}{l}\text { Beam extraction } \\
\text { efficiency }\end{array}$ & $90 \%$ & N/A & N/A \\
\hline $\begin{array}{ll}\text { Energy } & \text { spread } \\
\text { (typ) }\end{array}$ & $\pm 0.1 \%$ & $\pm 0.5 \%$ & $\pm 0.1 \%$ \\
\hline Energy stability & $\pm 0.1 \%$ & N/A & $\pm 0.1 \%$ \\
\hline
\end{tabular}

N/A Not (yet) available

IBA - Ion Beam Applications, Inc.

* While the energy extracted from the cyclotron is fixed, a variable thickness plastic wheel, which intercepts the beam, can reduce the energy to the desired level in a continuous fashion. The main penalties are higher emittance from multiple scattering and higher intensity losses from the energy degrading process which contribute to higher shielding requirements.

** AHAN - As High As Necessary. These intensities can exceed $100 \mu \mathrm{A}$ but they can be hardware limited at the ion source to the 30 to $300 \mathrm{nA}$ range.

*** Beam intensity uniformity for active beam scanning generally requires intensity regulating feedback loops to achieve uniform intensity. First demonstrated use of a scanning beam system occurred at the Berkeley synchrotron ${ }^{6}$ in 1992 . Adequate spill uniformity was achieved using intensity control feedback loops.

\section{SUMMARY}

The design effort for developing a hospital-based heavycharged-particle facility mandates a highly orchestrated program that requires the hospital personnel teams to work closely with the architects, physicists, and engineers of the vendors involved in the facility. The clinical beam delivery systems and the integrated control systems needed for the facility are complex and are, in general, less well developed than the accelerators, making an orchestrated development effort all the more necessary to ensure that all systems, including the accelerator, function for the benefit of the patient.

\section{ACKNOWLEDGEMENTS}

The authors wish to thank Optivus Technology, Inc., Yoshi Takada (Hitachi Corp.), Sashi Harada (Mitsubishi Electric Corp.), Luigi Picardi (ENEA, Frascati, Italy), and Jay Flanz (Massachusetts General Hospital, Boston, MA) for their valuable data regarding accelerator specifications at their institutions. Also, we wish to thank William Preston, Bob Knabenbauer, Stuart Wakefield, Chris Oeinck, CRA, and Joan De Paula, from Loma Linda University Medical Center, who helped to prepare this manuscript in its final form. Finally, we thank Daniel Miller, Loma Linda University Medical Center, for providing treatment planning data for this paper's presentation.

The authors take full responsibility for any inaccuracies which may have been reported here about the accelerators discussed. The reader is urged to contact the institutions directly for any accelerator questions which might arise.

\section{REFERENCES}

[1] J. Sisterson, Particles Newsletter, No. 23, Jan. 1999, Massachusetts General Hospital, Dept. Radiation Oncology, Boston, MA, Web site, http://www.neurosurgery.mgh. harvard.edu/hcl/ptles.htm

[2] J.M. Slater et al., "The Proton Treatment Center at Loma Linda University Medical Center", Int. J. Radiation Oncology, Biology and Physics, Vol. 22, p.383-389 (1992).

[3] J.F. Janni, "Proton Range-Energy Tables; 1KeV$10 \mathrm{GeV}$," Atomic and Nuclear Data Tables, Vol. 27, No.2-5, p.147-529 (1982).

[4] Private communications, A. Koehler, Harvard Cyclotron Laboratory, Cambridge, MA

[5] J. Debus et al., "Proposal for a Dedicated Ion Beam Facility for Cancer Therapy", Internal report, Gesellschaft fur Schwerionenforschung (GSI), Darmstadt, Germany.

[6] W.T. Chu et al., "Instrumentation in Medical Systems", Proceedings of the 1995 Particle Accelerator Conference, p.2394-2398, (1995). 